\title{
Lymphomatosis cerebri Presenting as a Recurrent Leukoencephalopathy
}

\author{
Floriane Courtois $^{a} \quad$ Michel Gille $^{b} \quad$ Frédéric Haven $^{c}$ \\ Philippe Hantson ${ }^{a}$ \\ ${ }^{a}$ Department of Intensive Care, Cliniques Universitaires Saint-Luc, and \\ Departments of ${ }^{b}$ Neurology and ${ }^{c}$ Radiology, Cliniques de l'Europe, \\ Université catholique de Louvain, Brussels, Belgium
}

\section{Key Words}

Primary central nervous system lymphoma $\cdot$ Lymphomatosis cerebri $\cdot$ Acute disseminated encephalomyelitis · Sentinel lesions $\cdot$ Steroids

\begin{abstract}
A 29-year-old immunocompetent woman was admitted in 2006 with ataxia, limb weakness, generalized dystonia, and vertical diplopia that developed after a febrile episode. Brain magnetic resonance imaging (MRI) revealed the presence of extensive periventricular white matter lesions that did not enhance after gadolinium injection. As low titers of cytomegalovirus-IgM antibodies were found in the serum, a presumed diagnosis of postviral acute disseminated encephalomyelitis (ADEM) was made, and the patient received a 5-day course of $1 \mathrm{~g}$ methylprednisolone. The clinical and radiological outcome was very rapidly favorable, and subsequent brain MRIs in 2007 and 2008 were normal. In March 2011, the patient was readmitted with the complaints of abnormal fatigue, imbalance, and speech disorder. The neurological examination showed fluctuating spatiotemporal disorientation with dyscalculia, verbal deafness, gait ataxia, right hemianopia, and pyramidal signs in the four limbs. The brain MRI demonstrated extensive $T_{2}$ hyperintense white matter lesions predominating in the left temporal and parieto-occipital lobes, with a pseudotumoral aspect enhancing with gadolinium contrast. A clinical improvement was transiently noted after pulse steroid therapy, but after relapse and radiological worsening, the diagnosis of recurrent ADEM was challenged. The brain biopsy confirmed the presence of primary central nervous system lymphoma (PCNSL) under the variant form of lymphomatosis cerebri. Despite a partial response to chemotherapy, the patient died 8 months after the diagnosis. We discuss the role of sentinel lesions that may precede PCNSL for several years and insist on the importance to consider early brain biopsy in the presence of extensive, nonenhancing white matter lesions, even in a young and immunocompetent patient.
\end{abstract}




\section{Introduction}

Primary central nervous system lymphoma (PCNSL) comprises less than $3 \%$ of all primary brain tumors [1]. Its mortality remains high despite chemotherapy and radiotherapy. In immunocompetent individuals, PCNSL usually appears on magnetic resonance imaging (MRI) as unique or multiple mass lesions with homogeneous contrast enhancement. Lymphomatosis cerebri (LC) is a rare variant of PCNSL, usually revealed by subacute dementia and gait disorders, and characterized on MRI by a diffuse infiltration of the cerebral white matter without any contrast enhancement at the early stage, and with frequent extension of the lesions to the brainstem, thalamus, and basal ganglia [2-5]. Such MRI features may mimic other white matter disorders such as gliomatosis cerebri, hypertensive encephalopathy, acute disseminated encephalomyelitis (ADEM), and other infectious and autoimmune encephalitides. We report on a patient who developed such a leukoencephalopathic variant of PCNSL more than 4 years after a presumed post-cytomegalovirus (CMV) ADEM.

\section{Case Presentation}

A 29-year-old immunocompetent woman presented with a subacute onset of gait ataxia, lower limb and right hand weakness, generalized dystonia, and transient vertical diplopia 3 weeks after a febrile episode in September 2006. The neurological examination also showed pyramidal signs and symptoms in the four limbs. Brain MRI revealed extensive lesions within the deep white matter, impinging on central grey nuclei together with subcortical involvement in frontal areas (fig. 1a, b). These lesions were more pronounced on the left side, appeared hyperintense on $\mathrm{T}_{2}$, FLAIR, and diffusion-weighted images, and did not enhance after gadolinium contrast injection. Low titers of CMV-IgM antibodies were found in the serum. The other serological investigations were negative, including Lyme disease, HIV, HSV, HZV, and EBV. The cerebrospinal fluid examination was normal without oligoclonal IgG bands. The diagnosis of ADEM following a possible recent CMV infection was considered. The patient was treated with a 5-day course of methylprednisolone $(1 \mathrm{~g} /$ day). The clinical course was rapidly favorable and the white matter lesions had completely disappeared on repeat MRIs in December 2006, March 2007, and April 2008. She remained asymptomatic until March 2011. At that time, she complained of abnormal fatigue, imbalance, and speech disorder. The neurological examination showed fluctuating spatiotemporal disorientation with dyscalculia, verbal deafness, gait ataxia, right hemianopia, and pyramidal signs in the four limbs. The cerebrospinal fluid examination was again normal, including the absence of oligoclonal IgG bands. The brain MRI demonstrated extensive $\mathrm{T}_{2}$ hyperintense white matter lesions within the parieto-occipital areas bilaterally, together with involvement of the splenium of the corpus callosum. Prominent mass effect was present on the left side as well as bilateral gadolinium-enhanced areas within the subependymal white matter of the

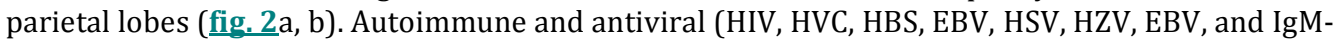
$\mathrm{CMV}$ ) serologies were negative. Methylprednisolone (1 g/day intravenously for 10 days) was prescribed for recurrent ADEM and, after significant clinical improvement, the patient left the hospital under decreasing doses of oral methylprednisolone. However, she was readmitted a few days later for spatiotemporal disorientation, headache, ataxia, and blurred vision. A brain MRI revealed a bilateral extension of the previously described lesions with involvement of the fronto-callosal areas. Pulse therapy with methylprednisolone $(1 \mathrm{~g} /$ day) was started again. She also presented with generalized tonic-clonic seizures and was transferred to the intensive care unit on April 7, 2011, with a Glasgow Coma Score of 8 (M5, V2, E1), a left hemiparesis, and a right third cranial nerve palsy. She was transferred to the university hospital for plasmapheresis. High-dose methylprednisolone therapy ( $1 \mathrm{~g} /$ day for 10 days) was combined to three courses of plasma exchanges. Epilepsy was controlled with valproate and levetiracetam therapy, and the patient regained consciousness with the same deficits as previously described. However, there was an extension of the lesions with gadolinium contrast enhancement. A stereotactic biopsy was performed on April 26, 2011, and it revealed a massive parenchymatous infiltration by large (CD20+) B lymphoid cells, showing numerous apoptotic bodies and mitotic figures. These cells were negative for GFAP, Olig2, CD10, and cycline D1 as well as for anti-CD21 and anti-CD23 antibodies. Aberrant membranous Bcl2 immunostaining and marked 
increased Ki67 proliferation index were found. In situ hybridization research for Epstein-Barr virus was negative. The diagnosis was primary CNS large B-cell lymphoma, and more particularly, its infiltrative variant termed LC. The neurosurgical procedure was complicated by a hematoma within the left frontal lobe with intracranial hypertension and coma. After a prolonged intensive care unit stay and four courses of chemotherapy (two with rituximab, methotrexate, vincristine, methylprednisolone, doxorubicin, and cyclophosphamide, and two with rituximab, methotrexate, and cytarabine), there was some neurological and radiological (fig. $3 \mathrm{a}, \mathrm{b}$ ) improvement, but new lesions appeared around the third ventricle. Radiotherapy was not considered after relapse as the patient remained in a poor neurological condition and palliative therapy was decided. The patient died 8 months after the biopsy.

\section{Discussion}

LC is a rare leukoencephalopathic variant of PCNSL that can be misdiagnosed, particularly in young immunocompetent individuals. Since the initial description of Bakshi et al. [6] in 1999, less than 20 cases have been described in the literature. In a recent review dealing with 16 patients, the mean patient age was 62.6 years at the time of diagnosis [7]. The outcome seems very poor as most reported patients died within 6 months after the onset.

Our patient had a biopsy-proven LC occurring more than 4 years after a first episode of acute leukoencephalopathy that had first been considered a possible post-CMV ADEM with a rapid clinical and neurological response to a 5-day course of high-dose methylprednisolone. However, the MRI findings were also consistent with LC, showing a diffuse involvement of the deep white matter extending to the brainstem and basal ganglia as well as to subcortical frontal areas. Considering the young age of the patient and her normal immune status, no brain biopsy was performed at this time. A first episode of LC could not be definitely ruled out, but seemed unlikely in regard to the prolonged remission.

In rare instances, PCNSL can coexist with $[8,9]$ or be preceded by demyelinating lesions with an interval ranging from several months to years [1,10-15]. For such tumefactive demyelinating lesions (TDL), Alderson et al. [12] have introduced the term sentinel lesions, which define contrast-enhancing focal lesions preceding the histopathological diagnosis of PCNSL by several months. These sentinel lesions may recede spontaneously or after corticosteroid treatment. They can also take the appearance of diffuse leukoencephalopathy. In the case published by Brecher et al. [13], radiological signs of diffuse leukoencephalopathy progressed over a period of 30 months. Interestingly, the lesions did not initially enhance after contrast administration, but well 9 and 24 months after initial presentation. The discrimination between TDL and PCNSL cannot be made on conventional MRI. Perfusion MRI and MR spectroscopy were not performed in our patient, but may potentially be helpful for the diagnosis of such lesions. On perfusion MRI, relative cerebral blood volume is usually reduced in TDL and increased in brain tumors such as PCNSL [16]. On MR spectroscopy, a similarly reduced NAA signal, an increased Cho signal, and increased Cho/NAA ratios are usually observed in both clinical entities, but increased glutamate/glutamine peaks are more consistent with TDL and not seen in an aggressive intracranial neoplastic process. Otherwise, elevated signals in the lipid region of the spectrum provide some clues to the diagnosis of PCNSL $[12,17]$. The definitive discrimination between PCNSL and TDL requires a brain biopsy. To our knowledge, 
there are no specific clinical, MRI, or pathological features in TDL that predict the development of PCNSL. Such lesions need careful monitoring and a repeat brain biopsy when there is no clinical or MRI response to immunosuppressive therapy.

The exact relationship between sentinel lesions and PCNSL is not well known. PCNSL should be masked when an immunosuppressive treatment is given prior to the first brain biopsy or kept dormant by host immunity. The presence of infiltrating lymphocytes expressing $\mathrm{T}$ lymphocyte markers in sentinel lesions is probably reflecting a cell-mediated immune response to the first signs of cerebral lymphoma in immunocompetent patients. This could explain the spontaneous disappearance of some of these lesions [12]. Lymphocytic infiltrates in sentinel lesions may also undergo transformation to monoclonal malignant B cell populations.

In conclusion, this case illustrates that the diagnosis of LC is a difficult challenge and cannot be made on conventional MRI alone. Although extremely unusual, this diagnosis should be considered in the presence of extensive, non-enhancing white matter lesions, even after the first episode of acute leukoencephalopathy, in a young and immunocompetent patient. Finally, the absence of an initial brain biopsy precluded the interpretation of the sentinel lesion that preceded LC.

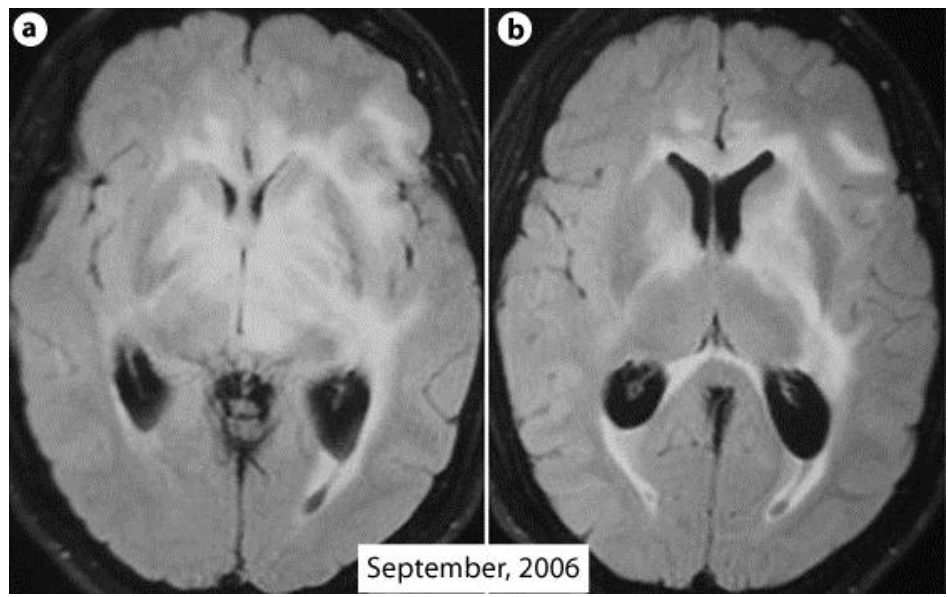

Fig. 1. September 2006. a, b Unenhanced FSE-FLAIR images with fat suppression option, showing diffuse abnormal hypersignal intensity within the deep white matter, impinging on central grey nuclei together with subcortical involvement in frontal areas. 

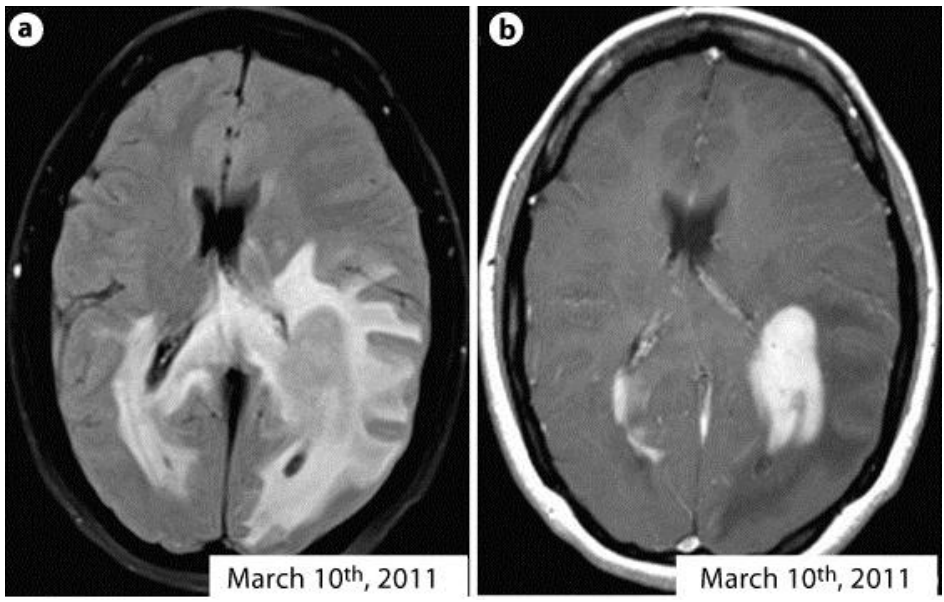

Fig. 2. March 2011. a, b Pretreatment (steroids and plasmapheresis) status on March 10, 2011. a Axial transverse FSE-FLAIR image, showing abnormal hypersignal intensity within parietooccipital areas bilaterally, together with involvement of the splenium of the corpus callosum. Prominent mass effect was present on the left side. $\mathbf{b}$ Contrast-enhanced axial transverse $\mathrm{T}_{1}$ weighted image, showing bilateral enhanced areas within the subependymal white matter of the parietal lobes.

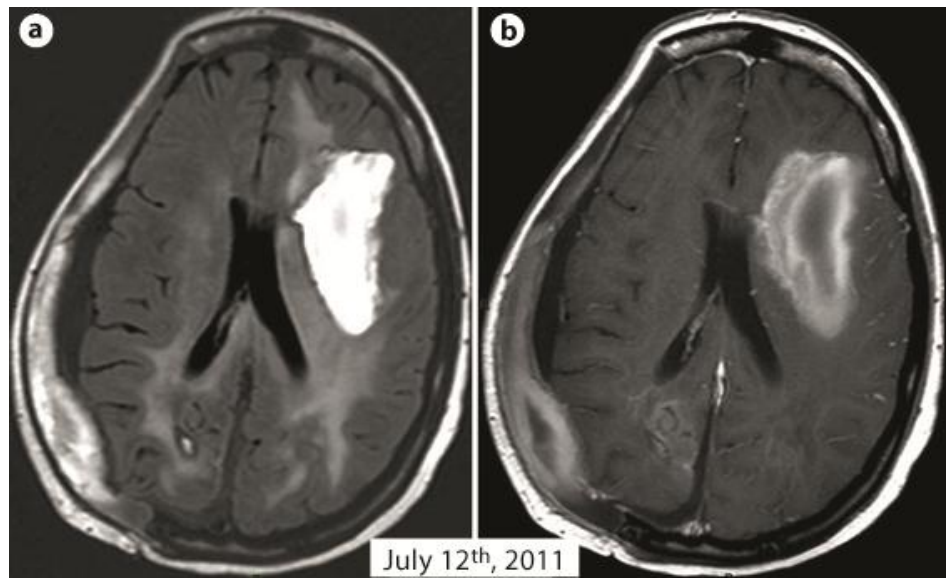

Fig. 3. July 2011. a Contrast-enhanced axial transverse FSE-FLAIR image, showing hemorrhage within the left frontal lobe and right parietal site of craniectomy. Hyperintense white matter changes were still present, but without any residual mass effect. $\mathbf{b}$ Contrast-enhanced axial transverse $\mathrm{T}_{1}$-weighted image in a similar slice location as in a, showing complete disappearance of tumor-like contrast enhancement. 


\section{References}

-1 Husseini L, Saleh A, Reifenberger G, Hartung HP, Kieseier BC: Inflammatory demyelinating brain lesions heralding primary CNS lymphoma. Can J Neurol Sci 2012;39:6-10.

-2 Rollins KE, Kleinschmidt-DeMasters BK, Corboy JR, Damek DM, Filley CM: Lymphomatosis cerebri as a cause of white matter dementia. Hum Pathol 2005;36:282-290.

-3 Lewerenz J, Ding X, Matschke J, Schnabel C, Emami P, von Borczyskowski D, Buchert R, Krieger T, de Wit M, Münchau A: Dementia and leukoencephalopathy due to lymphomatosis cerebri. J Neurol Neurosurg Psychiatry 2007;78:777-778.

4 Pandit L, Chickabasaviah Y, Raghothaman A, Mustafa S, Vasudevan A: Lymphomatosis cerebri - a rare cause of leukoencephalopathy. J Neurol Sci 2010;293:122-124.

-5 Yamamoto T, Kojima K, Koibuchi K, Ito S, Higuchi Y, Iwadate Y, Oide T, Kuwabara S: A case of primary central nervous system lymphoma presenting diffuse infiltrative leukoencephalopathy. Intern Med 2012;51:1103-1106.

-6 Bakshi R, Mazziotta JC, Mischel PS, Jahan R, Seligson DB, Vinters HV: Lymphomatosis cerebri presenting as a rapidly progressive dementia: clinical, neuroimaging and pathological findings. Dement Geriatr Cogn Disord 1999;10:152-157.

7 Kitai R, Hashimoto N, Yamate K, Ikawa M, Yoneda M, Nakajima T, Arishima H, Takeuchi H, Sato K, Kikuta $\mathrm{K}$ : Lymphomatosis cerebri: clinical characteristics, neuroimaging, and pathological findings. Brain Tumor Pathol 2012;29:47-53.

8 Bender GP, Schapiro RT: Primary CNS lymphoma presenting as multiple sclerosis. A case study. Minn Med 1989;72:157-160.

9 Burgetova A, Seidl Z, Vaneckova M, Jakoubkova M: Concurrent occurrence of multiple sclerosis and primary CNS lymphoma: a case report. Neuro Endocrinol Lett 2008;29:867-870.

10 Ruff RL, Petito CK, Rawlinson DG: Primary cerebral lymphoma mimicking multiple sclerosis. Arch Neurol 1979;36:598

11 Williams RS, Crowell RM, Fisher CM, Davis K, Lavyne MH, Ropper AH, Bremer AM: Clinical and radiologic remission in reticulum cell sarcoma of the brain. Arch Neurol 1979;36:206-210.

12 Alderson L, Fetell MR, Sisti M, Hochberg F, Cohen M, Louis DN: Sentinel lesions of primary CNS lymphoma. J Neurol Neurosurg Psychiatry 1996;60:102-105.

13 Brecher K, Hochberg FH, Louis DN, de la Monte S, Riskind P: Case report of unusual leukoencephalopathy preceding primary CNS lymphoma. J Neurol Neurosurg Psychiatry 1998;65:917920.

14 Kuhlmann T, Schroter A, Dechent P, Weber F, Rustenbeck HH, Füzesi L, Brück W, Ehrenreich H, Frahm J: Diagnosis of a multifocal B cell lymphoma with preceding demyelinating central nervous system lesions by single voxel proton MR spectroscopy. J Neurol Neurosurg Psychiatry 2001;70:259-262.

15 Ng S, Butzkueven H, Kalnins R, Rowe C: Prolonged interval between sentinel pseudotumoral demyelination and development of primary CNS lymphoma. J Clin Neurosci 2007;14:1126-1129.

16 Hakyemez B, Erdogan C, Yildiz H, Bozdogan E, Parlak M: Tumefactive demyelinating lesion: perfusionweighted imaging findings. EJR Extra 2005;53:95-98.

17 Cianfoni A, Niku S, Imbesi SG: Metabolite findings in tumefactive demyelinating lesions utilizing short echo time proton magnetic resonance spectroscopy. AJNR Am J Neuroradiol 2007;28:272-277. 